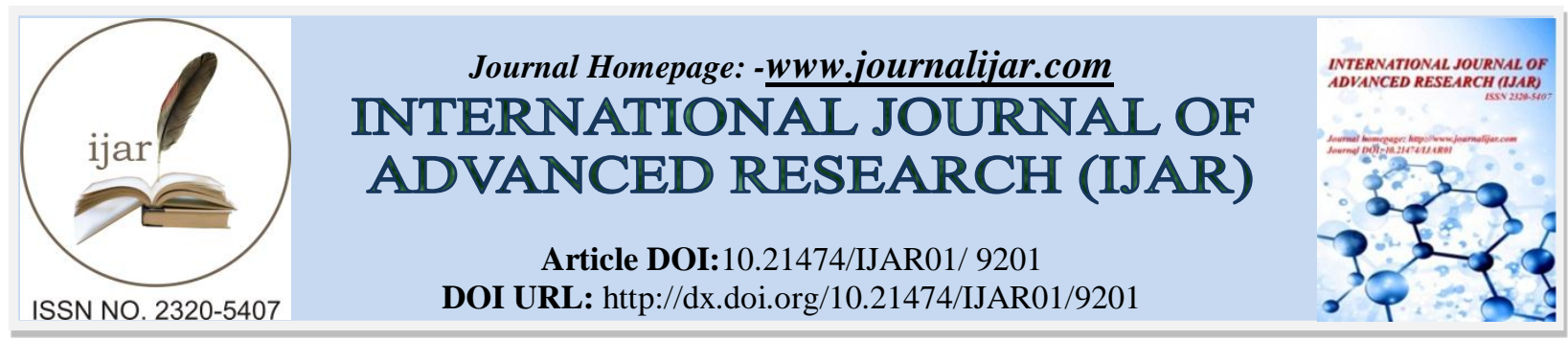

RESEARCH ARTICLE

\title{
LE PROJET PROFESSIONNEL DE L'ETUDIANT : UN DISPOSITIF PEDAGOGIQUE INNOVANT AU SERVICE DE L'INSERTION PROFESSIONNELLE.
}

Fatiha Benmimoun ${ }^{1}$, Mohamed Anouar Benaissa ${ }^{2}$ and Abdelhanine Belhaj ${ }^{3}$.

1. A. Belhaj, Professeur chercheur à la Faculté des Sciences de l'Education, Université Mohamed V, Rabat, Maroc.

2. M.A. Benaissa, Professeur chercheur, équipe : Sciences de l'ingénieur, sécurité et sciences entrepreneuriales (SIMoLab.net), CEDoc : Sciences et Techniques, Université Ibn Tofail, Kénitra, Maroc.

3. F. Benmimoun, Doctorante (PhD Student), équipe: Recherches Interdisciplinaires pour l'Innovation en Didactiques et en Capital Humain (RIIDCH), laboratoire : Analyse et Évaluation des Systèmes d'Éducation et de Formation, CEDoc: Homme, Société et Education, Faculté des Sciences de l'Education, Université Mohamed V, Rabat, Maroc.

\section{Manuscript Info}

Manuscript History

Received: 04 April 2019

Final Accepted: 06 May 2019

Published: June 2019

Key words:-

Student Professional Project, University Pedagogy, Pedagogical Innovation, Teaching Practices, Project Pedagogy, Professional Insertion, Competencies development.

\section{Abstract}

The professional project of the student (PPS) is presented as an innovative device proposed by the project pedagogy to mobilize the transversal skills of students, to integrate the initiatives of the actors around the concept of "project" and prepare them to discover the job market through a better orientation and the construction as they learn of a personal and professional project.

The PPS is also proving to be a promising alternative for both teachers and first-year university students (freshmore students), especially in the face of the various changes and challenges in higher education: the massive number of students, the heterogeneity of the public, and the rise of digital culture.

This article is the first part of an ongoing research on pedagogical innovations implemented in the context of teaching practices at the Moroccan university and its articulation with the professional skills required by the employer and guarantors of future employability.

It is based on a case study, put into practice for the benefit of freshmore students enrolled in Economics and Management (Ibn Tofail University, Kenitra). Our approach attempts to bring together the conceptual and practical framework for thinking about innovation in the programs, contents, methods and skills adopted during the implementation of the PPS and also through the activities of the students. We try to study academic innovation in a concrete way, through the design, implementation and evaluation of an innovative educational device.

Copy Right, IJAR, 2019,. All rights reserved.

\section{Introduction:-}

Alors que la pédagogie de projet propose une multitude de dispositifs, le projet professionnel de l'étudiant (le PPE)

Corresponding Author:-Fatiha Benmimoun.

Address:-Abdelhanine Belhaj, Professeur chercheur à la Faculté des Sciences de l'Education, 
semble émerger comme un dispositif innovant. Il s'agit d'une démarche pédagogique qui a déjà fait ses preuves puisqu'elle a été expérimentée dans un grand nombre de systèmes éducatifs (du primaire à l'universitaire), et ceci depuis le tout début du $20^{\text {ème }}$ siècle. L'intérêt renouvelé pour cette approche s'explique par les différentes possibilités qu'elle offre à la fois au professeur et à l'étudiant, tous deux avides de nouvelles connaissances. Le premier est à la recherche de meilleures pratiques enseignantes et d'aide à la construction des compétences de ses apprenants et le second est en quête de moyens d'apprentissage lui permettant une meilleure formation et une insertion professionnelle et sociale probante.

Ce dispositif pédagogique permet également de relier plusieurs disciplines, de mobiliser les compétences transversales des étudiants, et d'intégrer les initiatives des acteurs autour du concept «projet». Mais aussi, de préparer les étudiants à la découverte du marché du travail à travers une meilleure orientation et une construction au fur et à mesure de leur apprentissage, d'un projet personnel et professionnel qui pousse à l'exploration et la prospection (une sorte de visite virtuelle de l'au-delà du diplôme, et une projection dans un avenir professionnel dont les situations ne seront que complexes et variées).

Ceci dit, et avec la massification des effectifs étudiants (en tant que traduction chiffrée de la démocratisation de l'enseignement supérieur), associée à l'hétérogénéité des publics, et à l'évolution des pratiques d'apprentissage des étudiants (imprégnés notamment par la culture numérique), nous vivons aujourd'hui un bouleversement du paysage de l'enseignement supérieur qui n'a cessé de se transformer ces dernières années. Aussi, et tout comme les autres universités du monde, l'université marocaine est invitée à repenser ses différentes orientations et à revoir ses stratégies vis-à-vis des attentes de l'étudiant qui demeure « le cœur du système éducatif » et des objectifs visant à faire de lui un citoyen idéalement autonome, mais surtout prêt à l'emploi.

Face à ces évolutions et dans l'attente d'une réelle stratégie de rapprochement entre l'université et le monde de l'emploi, et qui soit mise en pratique dans les faits, voire obligatoire (comme pour certains systèmes éducatifs dans des pays tels que la France ou le Canada...), le PPE se révèle comme une solution alternative prometteuse et une piste à explorer à la fois par les enseignants et par les étudiants en première année à l'université.

Cependant, même si cette affirmation paraît évidente, elle soulève toutefois, de nombreuses interrogations face aux pédagogies universitaires mises en place et qui peinent à satisfaire les différentes attentes des employeurs futurs des lauréats de nos facultés (souvent recalés par ces recruteurs). Parmi les questions en suspens : comment les universités préparent-elles les étudiants à la vie professionnelle ? Est-ce au niveau des formations elles-mêmes, les rendant plus professionnalisantes, c'est-à-dire en rapprochant l'université du marché de l'emploi et en développant des compétences attendues par les employeurs ? Et si oui, cela peut-il s'appliquer à toutes les formations, y compris celles dites initiales? ...

Le présent article constitue le premier volet d'une recherche en cours sur les innovations pédagogiques mises en œuvre dans le cadre des pratiques enseignantes à l'université marocaine et son articulation avec les compétences professionnelles requises sur le marché du travail et permettant une employabilité au terme de la formation universitaire.

Basé sur une étude de cas, mise en pratique au profit des étudiants en première année de la filière «Sciences Economiques et de Gestion » de la Faculté des Sciences Juridiques Economiques et Sociales (Université Ibn Tofail, Kénitra), le présent travail rend compte d'une évaluation de la mise en place du dispositif pédagogique en question (à savoir, le PPE). Il apporte ainsi un éclairage sur des questions visant à savoir si les étudiants en première année universitaire, filière sciences économiques et de gestion, arrivent avec un projet professionnel ? Quel est concrètement l'impact de l'enseignement du PPE sur le projet professionnel de ces étudiants ? Quelles compétences ont été mises en œuvre lors de la réalisation de ce projet ? Quelles améliorations pédagogiques pourrions-nous mettre en place afin d'optimiser cet enseignement et le rendre davantage profitable aux étudiants ?

Après un bref aperçu du paysage universitaire marocain, nous procéderons, dans un premier temps, à une présentation du dispositif pédagogique du PPE ainsi qu'à l'évaluation de cet enseignement par les étudiants sondés. Par la suite, nous analyserons les différents résultats des évaluations en vue de développer cet outil par la formulation de propositions d'amélioration de ce dispositif pédagogique qu'est le PPE. 


\section{L'université marocaine et les perspectives d'insertion professionnelle}

L'enseignement supérieur national se donne comme objectifs principaux la formation et le développement des compétences ainsi que la diffusion des connaissances dans tous les domaines du savoir tout en contribuant au progrès scientifique, économique et professionnel, comme le stipule la loi 01-00 relative à l'organisation de l'enseignement supérieur ${ }^{[1]}$.

L'université est appelée ainsi à mettre en exergue ces prérogatives en renforçant son engagement académique et pédagogique et en prenant en compte les finalités professionnelles des formations dont la nature des savoirs enseignés est de plus en plus considérée comme devant répondre aux exigences du monde du travail ${ }^{[2]}$ (Monchatre, 2010).

Cette tendance vers la professionnalisation dans l'enseignement supérieur ne date pas d'aujourd'hui ${ }^{[3]}$ (Agulhon, 2007). Le processus éducatif se fixe également des priorités professionnelles en prévoyant des stages, des projets, des activités pratiques et autres ${ }^{[4]}$ (Rose, 2008). Il ne suffit pas d'offrir une solide formation au jeune diplômé, il faut qu'elle fasse de lui un candidat prêt à affronter le marché de l'emploi.

La notion d'employabilité, définie comme la capacité d'adaptation des futurs diplômés à des situations de travail variées ${ }^{[5]}$ (Corteel et Zimmermann, 2007), reste un défi pour l'université qui doit être relevé et dont elle doit tenir compte lors de la conception des programmes de formations.

Depuis plus d'une décennie, l'université marocaine a connu une série de réformes allant de la restructuration de sa gestion administrative à l'introduction du système LMD (Licence, Master, Doctorat) qui promet une fluide mobilité grâce aux passerelles, et remet profondément en cause ce que doit être un système de formation, sa qualité et surtout son adéquation aux véritables besoins du marché de l'emploi.

Le défi de l'insertion sociale et professionnelle reste, hélas, quasi-absent puisque l'offre pédagogique demeure confinée à sa vocation académique, en déphasage avec les exigences professionnelles et par conséquent avec la notion d'employabilité.

Les diplômés universitaires décrochent certainement un ticket d'entrée pour l'accès au marché de l'emploi mais sans pour autant pouvoir réellement y accéder. La raison est toute simple, tout l'investissement consenti en termes d'apprentissage et de formation s'articule autour des connaissances théoriques -dans la plupart des cas- dont l'excellente maîtrise ne garantit nullement une possibilité d'insertion.

Avec des curricula qui ne composent pas avec les référentiels de compétences et n'exigent point une suite logique prenant la forme de stage ou d'activités pratiques qui peuvent faciliter l'insertion professionnelle, la réforme universitaire s'est trouvée dans l'impasse. Pour corriger et comprendre les enjeux contradictoires qui sont à l'origine de cette impasse, une seconde réforme a vu le jour (dite plan d'urgence) afin de pallier le déficit de la première qui n'a pu atteindre tous ses objectifs, dont notamment celui de l'employabilité.

Le plan d'urgence (2009-2012), s'il a réussi relativement à rectifier le tir en rehaussant le taux de professionnalisation des filières de formation notamment dans les établissements universitaires à accès ouvert, il a introduit des passerelles imposées au niveau des écoles de commerce et d'ingénieurs (de l'ordre de 10\% chaque année). L'ouverture des filières sur l'interdisciplinarité et de l'université sur le monde socioprofessionnel a marqué cette étape (2009-2012) de réforme et a diffusé un certain espoir auprès des jeunes diplômés sensibles à la gravité de

1 Texte de la loi $\mathrm{n}^{\circ}$ 01-00 (du 19 mai 2000) portant organisation de l'enseignement supérieur au Maroc : www.enssup.gov.ma/sites/default/files/ETABLISSEMENT-SUP/Dahir_n-1-00-199_du19mai2000.pdf

2 MONCHATRE S., Du métier de sociologue au «genre sociologique »: réflexions sur la professionnalisation. Sociologies Pratiques, ${ }^{\circ} 21$, p. 139-147, 2010.

3 AGULHON C., La professionnalisation à l'université, une réponse à la demande sociale ? Recherches et Formations, $\mathrm{n}^{\circ}$ 54. 2007.

${ }^{4}$ ROSE J., La professionnalisation des études supérieures, tendances, acteurs et formes concrète. Les chemins de la formation vers l'emploi, Relief, 2008.

${ }^{5}$ CORTEEL D., Zimmermann B., Capacités et développement professionnel. Formation Emploi, n $98,2007$. 
l'alarme déclenchée par le haut-commissariat au plan. En effet, les statistiques (2016) indiquaient un taux de chômage de l'ordre de $25 \%$ guettant les porteurs d'un diplôme de niveau Bac +2 à Bac +3 et de $11 \%$ pour ceux ayant obtenu un diplôme de niveau Bac +5 et plus, notamment dans le cas des diplômés d'établissements universitaires à accès ouvert.

Car les contraintes politico-économiques et sociales, dont la nécessité d'assurer un diplôme au plus grand nombre d'étudiants (notamment avec la massification des effectifs) ont paradoxalement conduit à fixer aux premières années de l'enseignement universitaire, une double finalité (à niveau variable) priorisant des étudiants capables de poursuivre une formation supérieure avec succès, d'une part, tout en essayant de former des jeunes immédiatement employables dans des positions moyennes, d'autre part. De ce fait, l'une des priorités de ce plan d'urgence était essentiellement de doter le futur diplômé de compétences méthodologiques, linguistiques et communicationnelles pour augmenter ses chances d'insertion rapide dans le paysage socio-économique.

Ceci dit, il convient de rappeler que la formation universitaire est supposée contribuer davantage à la promotion des valeurs culturelles et éthiques sur lesquelles se basent l'identité nationale mais aussi celles que nécessitent la consolidation de la citoyenneté et du civisme tout comme stipulé dans la Charte Nationale d'Éducation et de Formation et conformément aux dispositions de la loi 01.00 portant sur l'organisation de l'enseignement supérieur. Les principaux apports de cette loi, par rapport à celles de 1975, résident dans l'autonomie pédagogique, administrative et financière de l'université, l'unité de l'université, l'ouverture de l'université sur la vie active, sur les autres établissements supérieurs de formation des cadres ou autres, mais aussi sur le monde socioéconomique. Sans oublier la mise au point d'une nouvelle architecture pédagogique qui se veut plus efficace de l'université ainsi que la restructuration et revalorisation de la recherche, la participation des étudiants dans la gestion des établissements et la mise en place de services sociaux aux étudiants.

Ces réformes, si novatrices soient-elles, n'ont pas réussi à remédier aux défaillances que connait l'université marocaine. Leurs limites font d'ailleurs l'objet de critiques soulignées par bon nombre de rapports récents, provenant d'instances internationales et nationales (UNESCO, Banque Mondiale, PNUD) et le dernier, celui du Conseil Supérieur d'Enseignement, dans sa vision stratégique de la réforme 2015-2030 et qui met l'accent sur les mesures prioritaires prévues dans l'actuel plan stratégique de la réforme du secteur de l'enseignement supérieur, de la recherche scientifique et de la formation des cadres.

Ces mesures mais aussi leurs critiques soulignent outre la nécessité d'une ouverture sur le monde socioprofessionnel, en tant que principal partenaire de l'université, l'importance du développement de l'offre de la formation universitaire, et l'aspect primordial du renforcement de la compétitivité des universités.

Et si, les Technologies de l'Information et de la Communication pour l'Enseignement (TICE), leur maîtrise et usage adéquat représentent un complément nécessaire susceptible de permettre à l'université de mieux gérer ses déficits en termes de taux d'encadrement qui ne cesse de baisser (départ en retraite des professeurs, et aucune politique claire pour le remplacement) et de capacité d'accueil inadaptée à l'effectif pléthorique qui croît exponentiellement. L'expérimentation d'une université virtuelle, en sus du développement et l'amélioration de celles physiques -ainsi que d'autres projets- s'avèrent relativement des options à considérer et à explorer. Mais, il convient surtout de garder à l'esprit que l'université virtuelle ne peut en aucun cas se substituer à celle physique de par la nature même de ce qui définit l'enseignement et l'apprentissage humain avec toutes ses dimensions tacites, d'interaction et d'observation inconcevables dans le contexte distant ou virtuel. D'où d'ailleurs l'échec du télétravail et toutes les recommandations en matière de gestion des connaissances qui vont évidemment à l'encontre de ce mode (distant) fût-il dans le contexte du travail (télétravail) ou de l'enseignement (e-learning ou université virtuelle).

En réalité, les motivations essentiellement économiques (de réduction des coûts et d'investissements, entre autres) peuvent difficilement concorder avec les exigences socioprofessionnelles (de leadership, management d'équipe, gestion des conflits, conduite du changement, et autres situations à vivre et à gérer en groupe et en présentiel -c'està-dire sur site, au sens lieu d'apprentissage et/ou de travail commun- et non par écrans interposés ou géographiquement éparpillés), et encore moins avec les finalités pédagogiques et d'excellence académique.

D'ailleurs, l'actuelle vision stratégique de l'enseignement supérieur marocain souligne justement le rôle capital que joue l'Agence Nationale d'Evaluation et d'Assurance Qualité de l'Enseignement Supérieur et de la Recherche Scientifique (créée en 2015). Agence qui a vu le jour en réponse aux exigences précitées et aux besoins d'assurance 
qualité. Une structure qui a entre autres pour vocation, l'amélioration des dispositifs de formation. Parmi ses missions les plus prioritaires, l'élaboration de mécanismes permettant la valorisation de la culture de l'évaluation et de la qualité au sein de l'université marocaine, la mise en place d'un référentiel d'évaluation et d'assurance qualité, et la révision des critères d'accréditation des filières de formation, en faisant de l'insertion professionnelle une priorité. Ainsi, toute filière qui n'assure pas de débouchés pour les étudiants, futurs demandeurs d'emploi ne sera plus reconduite ${ }^{[6]}$.

D'une manière générale, l'enseignement (avec tous ses niveaux) s'est toujours placé comme un chantier prioritaire dans la politique publique nationale. Nous rappelons que la démocratisation de l'enseignement supérieur exige de l'université marocaine, une meilleure prise en compte de l'employabilité et de l'insertion professionnelle de ses étudiants. Une obligation soulignée par le système LMD, une nécessité économique et un impératif social. Il en résulte l'obligation de doter les étudiants de compétences fonctionnelles pour accéder aux métiers mais aussi de leur proposer des formations ayant des débouchés clairement identifiés, leur permettant une insertion rapide, efficace et en corrélation avec leurs compétences et aspirations.

L'université se voit -au terme de cet état des lieux synthétique- soumise à de fortes pressions tant internes qu'externes. Avec d'une part, des rapports nationaux et internationaux, faisant toujours et encore état de taux de chômage alarmant parmi les diplômés universitaires et engendrant moult réactions, manifestations et réflexions allant de pair avec ces rapports, et qui soulignent de manière récurrente l'inadaptation de l'offre universitaire aux attentes du monde de l'entreprise, sans mettre le doigt ou préciser les causes profondes de cet état des choses. Une inadéquation qui donne lieu évidemment à des réformes mais qui, à chaque fois, espèrent se positionner comme des palliatifs préconisés, sans s'intéresser à la question de la « pédagogie universitaire » qui demeure le parent pauvre de ce débat qui fait pourtant rage. Omise, inexplorée ou peu explorée, nous constatons, effectivement, que cette dernière est rarement évoquée dans ces réformes ou rapports.

A croire que les pratiques enseignantes dans l'enseignement supérieur sont à la marge de toute réflexion sur le devenir de l'université. D'ailleurs, la rareté des recherches en pédagogie universitaire confirme ce constat surprenant.

C'est pourquoi, dans cette contribution nous essayerons, sans aucune prétention à l'exhaustivité, de cerner l'essentiel des nouvelles pratiques à l'université quant à leur articulation avec le double objectif de formation et d'insertion professionnelle. En effet, différentes méthodes ont le vent en poupe au sein des universités, telles les pédagogies actives en particulier, auxquelles s'ajoute l'élaboration de référentiels de compétences (inspirés de ceux des branches professionnelles concernées), ce qui permet une mise en situation professionnelle des étudiants et un développement de leurs compétences professionnelles. Dans le contexte que nous avons décrit, les pratiques enseignantes sont supposées changées pour accompagner tous les remous que connait le paysage universitaire en général et marocain notamment.

\section{La pédagogie de projet et les innovations pédagogiques Pédagogie de projet :}

La pédagogie de projet fait partie des pédagogies actives et renvoie à un modèle d'apprentissage lié aux modèles cognitivistes, constructivistes, et socio-cognitivistes ${ }^{[7]}$ (Abdallah, 2009). Cet apprentissage par projet s'inspire essentiellement d'une conception socioconstructiviste de l'apprentissage qui selon plusieurs auteurs nécessiterait une pédagogie différenciée qu'ils prônent en lieu et place de toute forme traditionnelle d'enseignement.

Pour Perrenoud, cette démarche de pédagogie par projet n'est autre qu'un simple exercice où il considère le projet comme une expérience de contextualisation qui confronte les apprenants à des obstacles et provoque des situations d'apprentissage sans qu'il soit foncièrement une fin en soi. Toutefois, si les apprenants excellent lors de cette expérience, la réussite du projet devient un enjeu fort, et tous les acteurs, professeurs et apprenants, sont tentés de

\footnotetext{
${ }^{6}$ Entretien avec le directeur de l'ANEAQ (28/02/2017) : http://www.aneaq.ma/fr/aneaq_eco.pdf.

${ }^{7}$ ABDALLAH F., Meta-modélisation pour décrire et instrumenter une situation d'apprentissage de pédagogie par projet collectif. Thèse de doctorat en informatique. Le Mans : Université du Maine, 2009.
} 
viser l'efficacité au détriment des occasions d'apprentissage. Pour apprendre, il faut que chaque acteur soit mobilisé, accepte la contradiction, la travaille, et l'anticipe. C'est ce qui garantit une "double dévolution" ${ }^{8]}$.

Il est clair que cette démarche repositionne l'apprenant en tant qu'acteur et auteur de son apprentissage en invitant le professeur à créer une dynamique interactive dans l'enseignement. Un tel écosystème favorise d'abord l'épanouissement personnel de l'apprenant, sa confiance en ses habilités et la fierté devant ses réalisations. Ensuite, il importe de souligner que son impact positif dépasse de loin un sentiment de fierté ou d'aisance affective, pour influencer également l'apprentissage des apprenants et le développement des compétences professionnelles. L'approche par projet se définit comme l'ensemble des activités de résolution d'un problème issu de la vie réelle. Ces activités s'inspirant des méthodes tantôt intuitives et tantôt réflexives. "Intuitives » puisqu'elles mettent en œuvre une démarche d'anticipation et «Réflexives » quand elles poussent l'apprenant à réfléchir à la résolution du problème tout en respectant un planning précis. Leur résultat est double : d'une part, une production concrète, matérielle ou intellectuelle et d'autre part, le développement chez l'apprenant de savoirs de haut niveau ainsi que de compétences transversales ${ }^{[9]}$ (Proulx, 2004)

Il est à rappeler que le projet doit placer l'apprenant dans une situation réelle, proposant un défi à relever ${ }^{[10]}$ (Lebrun, 2007), dont la concrétisation demeure viable et évaluable (Perrenoud, 2002 ; Proulx, 2004 ; Abdallah, 2009). Au cours de cette expérience, l'enseignant ne peut être que motivateur et médiateur pour laisser plus de liberté d'action à l'apprenant qui devient acteur et auteur de son apprentissage.

En sus des savoirs, des savoir-faire se produisant dans l'action, ce type d'apprentissage développe des compétences transversales ${ }^{[11]}$ (Le Boterf, 2003) et d'autres attitudes telles que l'autonomie et la responsabilité. Il favorise également le travail en équipe, l'ouverture et la polyvalence.

Les fondements théoriques s'inspirent également de l'approche éducative en orientation qui a été développée dans les années 1970 et 1980 par des chercheurs comme Latreille ${ }^{[12]}$, qui considère que l'individu peut avoir une prise sur le cours de sa vie, qu'il a la possibilité de travailler par lui-même son projet personnel et professionnel, aux fins de réaliser des choix éclairés par la mobilisation de l'expérience. Ces pratiques enseignantes innovantes ont non seulement une certaine incidence sur l'insertion professionnelle mais aussi sur la réussite universitaire. Galand, Neuville et Frenay (2005) supposent que l'échec universitaire des étudiants en premier cycle pourrait bien être lié aux pratiques d'enseignement ${ }^{[13]}$. Romainville et Parmentier (1998) ajoutent que « les procédures que les étudiants déclarent mettre en œuvre à l'occasion d'un cours sont influencées par la méthode pédagogique de l'enseignant » [14] . L'amélioration de la pédagogie universitaire pourrait même constituer une véritable solution pour lutter contre l'échec, puisque cela reviendrait essentiellement à «privilégier les méthodes qui suscitent l'apprentissage en profondeur » et à «revaloriser la mission d'enseignement». Ce postulat émis par Leroux (1997) et repris par Romainville (2000) considère la pratique du cours magistral comme étant « inscrite dans la tradition universitaire » et constituant «un facteur d'échec en premier cycle».

\section{L'intérêt du PPE}

Dans le contexte marocain, la plupart des bacheliers se trouvent parachutés dans le monde universitaire sans aucune visibilité ni académique, ni professionnelle.

${ }^{8}$ PERRENOUD P., Réussir ou comprendre ? Les dilemmes classiques d'une démarche de projet, Genève.1998.

${ }^{9}$ PROULX J., Apprentissage par projet, Presses de l'Université du Québec, 2004.

${ }^{10}$ LEBRUN M., Théories et méthodes pédagogiques pour enseigner et apprendre : Quelle place pour les TIC dans l'éducation? (2e éd.). Bruxelles : De Boeck Supérieur. 2007.

${ }^{11}$ LE BOTERF G., Compétence et navigation professionnelle. Paris : Éditions d'Organisation. 2000.

${ }^{12}$ LATREILLE G., Les chemins de l'orientation professionnelle : trente ans de lutte et de recherche. Lyon : Presses Universitaires de Lyon. 1984.

${ }^{13}$ GALAND B., Neuville, S. et Frenay, M., L'échec à l'université en Communauté Française de Belgique : Comprendre pour mieux prévenir ? In Galand B. (dir.) : L'échec à l'université en Communauté Française de Belgique (pp. 5-17). Cahiers de recherche en éducation et formation, $n^{\circ} 39.2005$.

${ }^{14}$ ROMAINVILLE M. et Parmentier, P., Les manières d'apprendre à l'université. In Frenay M., Noël B., Parmentier P. et Romainville M. (dir.) : L'étudiant-apprenant : Grilles de lecture pour l'enseignant universitaire (pp. 63-80). Bruxelles : De Boeck Université. 1998. 
En effet, et en l'absence totale d'une quelconque orientation universitaire ou même scolaire, le plus souvent, l'étudiant est livré à lui-même et la perspective de son insertion professionnelle n'apparait point comme un impératif à prendre en considération.

Depuis quelques années, nous avons jugé utile d'implanter un dispositif de formation, d'une dizaine d'heures, au profit de nos étudiants, et réparties comme suit : d'une part, une première séance dédiée à la présentation générale mais détaillée de ce dispositif (sous la forme d'un cours magistral) et d'autre part, des séances d'encadrement et de suivi. Il s'agit du Projet Professionnel de l'Étudiant (PPE), mis en place par nos soins dans le cadre du cours que nous assurons et qui est intitulé : «Langue et Terminologie Economique (LTE)». Lequel relève du cursus des étudiants de première année, et plus précisément, en second semestre.

L'objectif de ce dispositif est d'abord de sensibiliser les étudiants à la question du devenir professionnel par un exercice de recherche en petits groupes, consistant dans un premier temps à réunir des informations relatives au métier d'avenir (de leurs choix) ou encore, pour ceux qui ont une certaine préférence pour l'entrepreneuriat, au projet de création de leurs propres entreprises. Et, dans un second temps, une fois ces informations recueillies (en ligne grâce aux moteurs de recherche ou hors ligne auprès des centres de documentation et des professionnels, via des rencontres et entrevues), elles doivent être présentées oralement et consignées par écrit dans un rapport. Ce type de pratiques enseignantes innovantes permet à l'apprenant de consolider son nouveau statut d'étudiant (qui se doit d'apprendre à demander, par opposition à son ancien statut d'élève où il recevait pour l'essentiel), de s'adapter davantage à cette nouvelle culture universitaire mais également de retrouver un point d'ancrage, d'affiliation et d'intégration à l'université (Coulon, 1997) ${ }^{[15]}$.

Le PPE ne s'avère pas seulement un marqueur de transition (dans le temps et dans l'espace) du lycée vers l'université, mais se révèle être également l'opportunité pour ces étudiants de développer de nouvelles compétences relatives à l'organisation de leur travail individuel et en équipe, à la prise de parole en public et aux techniques d'animation entres autres. Des compétences qu'ils n'ont que rarement l'occasion d'expérimenter dans les éventuelles autres activités qu'ils pourraient se voir faire-faire sur le lieu de leur formation universitaire. Puisque, pour les enseignants aussi, le positionnement par rapport au savoir et par rapport aux étudiants, la nature des interactions qu'ils peuvent avoir avec ces derniers, les tâches qu'ils doivent assurer et les outils qu'ils sont amenés à utiliser sont tous transformés par ce type de pédagogie que l'on pourrait qualifier de « proactive ».

Ce dispositif «Projet Professionnel de l'Étudiant » vise également une amélioration de l'orientation professionnelle chez les étudiants inscrits en première année, en sciences économiques et de gestion (dans notre cas) en faisant en sorte que chaque étudiant puisse se mettre à réfléchir en mode projet. Objectif qui, au vu des évaluations anonymes réalisées auprès de ce public, semble être atteint.

\section{Le PPE : Un dispositif innovant ?}

Pour mieux cerner cette question, nous nous référons aux différents travaux de Béchard et Pelletier qui considèrent que dans le « contexte universitaire, les innovations pédagogiques sont souvent décrites comme tout ce qui ne relève pas de l'enseignement magistral, méthode encore utilisée par une très grande majorité de professeurs. » ${ }^{[16]}$ (Béchard \& Pelletier, 2001). Ces mêmes auteurs considèrent l'innovation « pédagogique » dès lors qu'elle « cherche à améliorer substantiellement les apprentissages des étudiants en situation d'interaction et d'interactivité ».

En effet, le caractère novateur ou innovant des pratiques enseignantes et des méthodes d'apprentissage exige de l'apprenant une implication particulière et une interaction, lesquelles favorisent la compréhension et l'application des connaissances. Caractère qui nécessiterait des concepteurs de programmes universitaires une meilleure prise en compte des exigences professionnelles et des attentes des recruteurs en privilégiant davantage la contextualisation des connaissances.

${ }^{15}$ COULON A., Le Métier d'étudiant : l'entrée dans la vie universitaire. In: Revue française de pédagogie, volume 131. Les formations professionnelles entre l'Ecole et l'Entreprise. pp. 138-139. 2000.

${ }^{16}$ BECHARD J.-P. et Pelletier P., Développement des innovations pédagogiques en milieu universitaire : un cas d'apprentissage organisationnel, in Raymond D. (dir.), Nouveaux espaces de développement professionnel et organisationnel, Éditions du CRP, 2001. 
Ceci dit, il importe de rappeler que l'interaction des apprenants est à concevoir dans un cadre collectif, tout comme l'ont souligné certains travaux, du fait que «le savoir est construit dans une histoire collective qui est celle de l'esprit humain et des activités de l'homme, et il est soumis à des processus collectifs de validation, de capitalisation, de transmission. [...] Aussi, les rapports de savoir sont-ils, plus largement, des rapports sociaux. » ${ }^{[17]}$ (Charlot, 1997).

Il est à noter que l'"innovation", sous l'angle de la pédagogie, requiert des conceptions qui changent selon les contextes et les postures des pédagogues ${ }^{[18]}$. Les chercheurs l'abordent à travers plusieurs aspects, tels que : les dispositifs, l'apprentissage, les programmes, les compétences, l'évaluation, etc. Et ce, dans une perspective privilégiant (la mesure de) l'efficacité (en recourant à une batterie d'outils d'analyses) de l'action pédagogique.

Dans ce sens, Cros et Adamczewski ${ }^{[19]}$ perçoivent l'innovation comme « une démarche d'implantation d'une novation dans un milieu donné, différent de celui d'où elle origine ». Autrement dit, les auteurs qualifient d'innovations, toutes les situations dans lesquelles les enseignants, dans leur établissement, mettent en place en les adaptant des méthodes déjà connues, comme : les apprentissages par projets et par problèmes, les classes inversées, les recherches collaboratives, les outils numériques ou les technologies éducatives, etc.

L'innovation pédagogique est souvent perçue, à tort, seulement sous l'angle de la technologie. Certes, cette dernière propose de nouvelles manières (et/ou moyens) d'enseigner, conséquence naturelle de la révolution technologique, et de la multiplication des outils numériques, dont l'impact s'avère pertinent ${ }^{[20]}$, selon certains auteurs.

Une perception corrigée ou complétée par des spécialistes de la question de «l'innovation pédagogique » comme Béchard (2001) ${ }^{[21]}$ par exemple, dans sa recherche de la définition de l'innovation et qui a soulevé le caractère (naturellement) confus et indifférencié qui allie tantôt les innovations technologiques, tantôt les innovations curriculaires et parfois même les innovations pédagogiques. D'où l'encouragement de ce couplage de l'activité et de l'interactivité des étudiants, à savoir : en classe et hors classe, au détriment de l'enseignement magistral (qui lui reste associé essentiellement à la pédagogie passive ou transmissive).

Une conception de l'innovation pédagogique qui va dans le sens des pédagogies actives, lesquelles s'inspirent quant à elles d'une conception constructiviste des apprentissages, et s'imposent désormais comme un modèle célébrant l'autonomie des étudiants vis-à-vis des situations d'apprentissage.

Notre démarche, dans cet article, vise à rapprocher les cadres conceptuel et pratique pour penser l'innovation tant à l'échelle du curriculum, des contenus, des méthodes et des valeurs que des activités des étudiants. Nous cherchons à examiner l'innovation en milieu universitaire de manière tangible, à travers la conception, le développement, l'implantation et l'évaluation d'un dispositif pédagogique, innovant par conséquent.

La pédagogie par projet -en tant que pratique relevant de la pédagogie active- offre en sus de ce cadre générateur d'apprentissages qu'est la réalisation d'une production concrète (à l'instar du projet professionnel de l'étudiant), une réponse adaptée également aux nouvelles exigences des étudiants universitaires, friands de méthodes actives et consommateurs par excellence de tout ce qui représente une rupture avec le passé et avec le pouvoir de l'inertie. En effet, les programmes d'apprentissage par problèmes ou par projet s'inscrivent dans cette perspective (dynamique) puisqu'ils amènent habituellement les étudiants à développer des compétences professionnelles qui repositionnent la

${ }^{17}$ CHARLOT B., Du rapport au savoir. Éléments pour une théorie. Paris : Anthropos. 1997.

${ }^{18}$ LISON C., Bédard D., Beaucher C. et Trudelle D., De l'innovation à un modèle de dynamique innovationnelle en enseignement supérieur. Revue Internationale de Pédagogie en Enseignement Supérieur (RIPES), 30-1, 2014. En ligne : http://ripes.revues.org/771.

${ }^{19}$ CROS F. et Adamczewski, G. (dir.), L'innovation en éducation et en formation. Bruxelles, De Boeck.1996.

${ }^{20}$ POUMAY M., L'innovation pédagogique dans le contexte de l'enseignement supérieur. In: Lameul G. et Loisy C. (dir.). La pédagogie universitaire à l'heure du numérique (p. 69-81). Louvain-la-Neuve : De Boeck. 2014.

${ }^{21}$ BECHARD J.-P., L'enseignement supérieur et les innovations pédagogiques : une recension des écrits. Revue des sciences de l'éducation, 272 (2001), 257-281. 2001. 
valeur de vérité des savoirs en fonction de situations problèmes auxquelles ils sont attachés (rapports contextuels) [22]

Le projet professionnel de l'étudiant (PPE) est une pédagogie qui vise l'orientation [23] (Latreille, 1984) vers l'emploi et qui montre que la construction d'un projet professionnel est un déterminant essentiel de la motivation et de la réussite des étudiants. Ces nouvelles missions d'accompagnement à la réussite et à l'insertion professionnelle dévolues désormais à l'université, du moins en France, et par voie de conséquence aux enseignants, modifient profondément la nature même de la pratique enseignante.

La réussite de l'implantation de ce dispositif (PPE) ou tout autre dispositif pédagogique innovant nécessite un engagement à la fois du corps enseignant et administratif puisque sa mise en place demande une organisation à la fois pédagogique et administrative.

La conjugaison des savoirs académiques et professionnels se fait nécessairement en fonction du projet de l'étudiant. Pour atteindre un tel objectif, seul un accompagnement personnalisé permet d'aborder les compétences individuelles de chaque étudiant. Une démarche qui s'avère coûteuse pour les enseignants engagés dans cette voie : un investissement en termes de temps (imparti aux séances d'encadrement), des approches pédagogiques sur mesure, une organisation matérielle particulière (la disponibilité des locaux, des listes d'étudiants actualisées, mais encore celle des étudiants eux-mêmes), entre autres.

Ceci dit, les retours sur ce type d'investissement s'avèrent, in fine, plus que concluants. En effet, et comme on le verra, le dispositif déployé, auprès de plusieurs groupes, a été évalué anonymement par les étudiants et les résultats, ci-après, révèlent leur appréciation du dispositif pédagogique PPE, tout comme le relatent leurs points de vue sur les apprentissages réalisés.

\section{Implantation et évaluation du PPE \\ Démarche de la recherche}

L'étude vise à comprendre et à expliquer l'incidence que peut avoir cette innovation pédagogique qu'est le PPE sur la préparation à l'insertion professionnelle des étudiants ayant suivi cette formation.

La compréhension des processus induits par le projet professionnel de l'étudiant, a été rendue possible non seulement par voie de questionnaire, mais aussi par divers observations et échanges avec les étudiants, tout au long de leur réalisation du PPE, aussi bien au niveau des choix d'orientation que de leurs modifications (de ces choix). Sans oublier, les réflexions et interactions suscitées ou enclenchées à l'occasion de l'introduction de ce dispositif en séance magistrale et visant à amorcer cette démarche de recherche. Ce qui a permis, dès le départ, un premier recueil de données, auprès de ce public étudiant. Nous nous sommes également servis des productions qui ponctuent le déroulement du dispositif : fiches réalisées au moment du choix de la vocation professionnelle, supports de la présentation finale mais aussi recommandations et mises au point ponctuelles avec les équipes étudiantes en charge de ce PPE au fur et à mesure de leur réalisation de ce dernier.

Enfin, nous avons développé une analyse qualitative de ces productions, écrites et orales, afin de repérer d'éventuelles phases de construction de ce projet et en vue d'identifier ce qui peut entraver cette élaboration du PPE. Aussi, notre méthodologie s'est-elle appuyée tant sur des questionnaires que sur des entretiens semi-directifs, effectués directement auprès des groupes en charge du PPE (en face à face et au format papier). L'approche inductive nous a permis en outre la mise en valeur non seulement du caractère qualitatif des données recueillies mais également de mettre davantage l'accent sur les compétences essentielles à un métier. Cette approche se veut donc « un ensemble de procédures systématiques permettant de traiter des données qualitatives, ces procédures étant essentiellement guidées par les objectifs de recherche. Elle s'appuie sur différentes stratégies utilisant prioritairement la lecture détaillée des données brutes pour faire émerger des catégories à partir des interprétations

\footnotetext{
${ }^{22}$ LISON C et Bédard D., Développement de la posture intellectuelle d'étudiants universitaires dans un programme innovant, Revue internationale de pédagogie de l'enseignement supérieur, 30-1, 2014. http://ripes.revues.org/795

${ }^{23}$ LATREILLE G., Les chemins de l'orientation professionnelle : trente ans de lutte et de recherche. Lyon : Presses Universitaires de Lyon. 1984.
} 
du chercheur qui s'appuie sur ces données brutes » ${ }^{[24]}$ (Blais, Martineau, 2006). L'analyse inductive fait ainsi ressortir les dimensions encore inexplorées de la problématique visée.

Le dispositif PPE permet aux étudiants de cerner la facette réelle de l'activité professionnelle choisie (Clot, 1999) et de resituer leurs parcours et leurs projets dans le cadre d'une " activité constructive » (Pastré, 2011). Ainsi, l'étudiant engagé dans cette activité pédagogique réussit non seulement à appréhender la complexité du réel mais aussi à saisir l'importance de la connaissance de soi (à travers un bilan personnel) -une connaissance, souvent négligée en faveur d'une identification au groupe-dans une démarche d'engagement professionnel.

Rappelons que si l'objectif de ce dispositif, à savoir le projet professionnel de l'étudiant (PPE) est d'aider les étudiants à mieux se connaître et identifier leurs compétences, il offre également l'occasion à ces étudiants, en première année à l'université, de reconsidérer sérieusement la question du devenir professionnel. Cette expérience se veut donc un moment (critique) de vérité, amenant les étudiants dès lors à valider ou assumer pleinement le choix de leur orientation professionnelle.

\section{Échantillon d'enquête, recueil et analyse des données par questionnaires papier}

A partir d'une population d'un peu plus d'un millier d'étudiants, en première année, inscrits en sciences économiques et de gestion (à la Faculté des Sciences juridiques, économiques et sociales, relevant de l'université Ibn Tofail de Kénitra, Maroc), qui ont tous bénéficié de ce dispositif déployé et assuré par nos soins, nous en avons invité plus de 350 (étudiants) à exprimer leur appréciation (de ce dispositif) via des questionnaires papier se présentant sous la forme d'une double feuille $\mathrm{A} 4$, avec des questions en double colonnes, aussi bien des questions fermées que ouvertes.

Nous avons réussi à exploiter 278 réponses valides (avec une marge d'erreur de $5 \%$ et un niveau de confiance de 95\%). Il est à noter que la programmation de ce dispositif a suscité une implication remarquable à la fois des étudiants et de l'enseignant que nous sommes. Ce qui a permis un taux de réponse de plus de $80 \%$.

Notre hypothèse principale suppose que l'implantation du PPE dans la formation universitaire et au profit notamment des étudiants de la première année est à même d'offrir l'occasion à ces jeunes recrues de l'université de reconsidérer leur devenir professionnel, de le structurer sous forme de projet. Et, éventuellement de recadrer ou corriger l'orientation académique de l'étudiant de manière à développer en lui davantage les compétences correspondant à sa réorientation, s'il y a lieu, pour permettre son insertion professionnelle future.

Qui sont-ils ? (Ventilation de la population sondée par sexe)

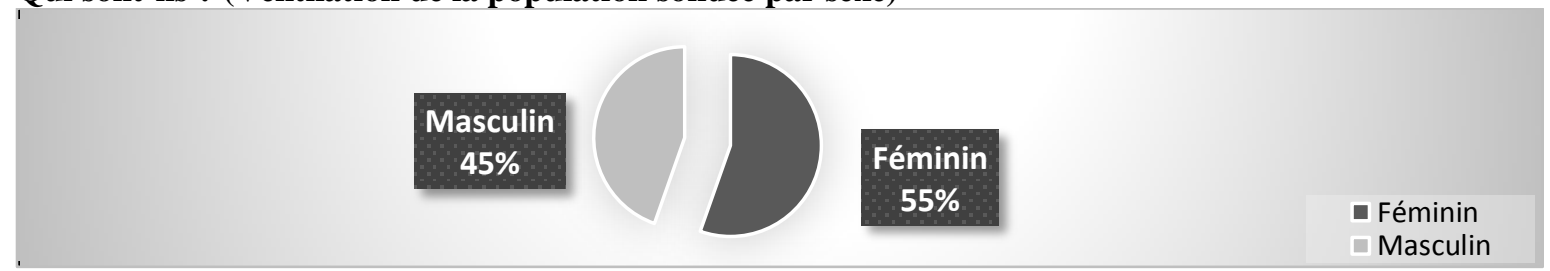

Figure 1:-Répartition par sexe

La population totale des répondants se compose de 278 répondants, dont $55,4 \%$ sont de jeunes étudiantes et $44,6 \%$ sont de jeunes étudiants. La répartition hommes/femmes fait ressortir une quasi-parité.

Nous précisions également que $92 \%$ des répondants sont de nationalité marocaine et $8 \%$ représentent des nationalités étrangères.

\footnotetext{
${ }^{24}$ BLAIS M. et Martineau S., L'analyse inductive générale : description d'une démarche visant à donner un sens à des données brutes, Recherches qualitatives, vol. 36, n², 2006.
} 


\section{Les origines scolaires des sondés}

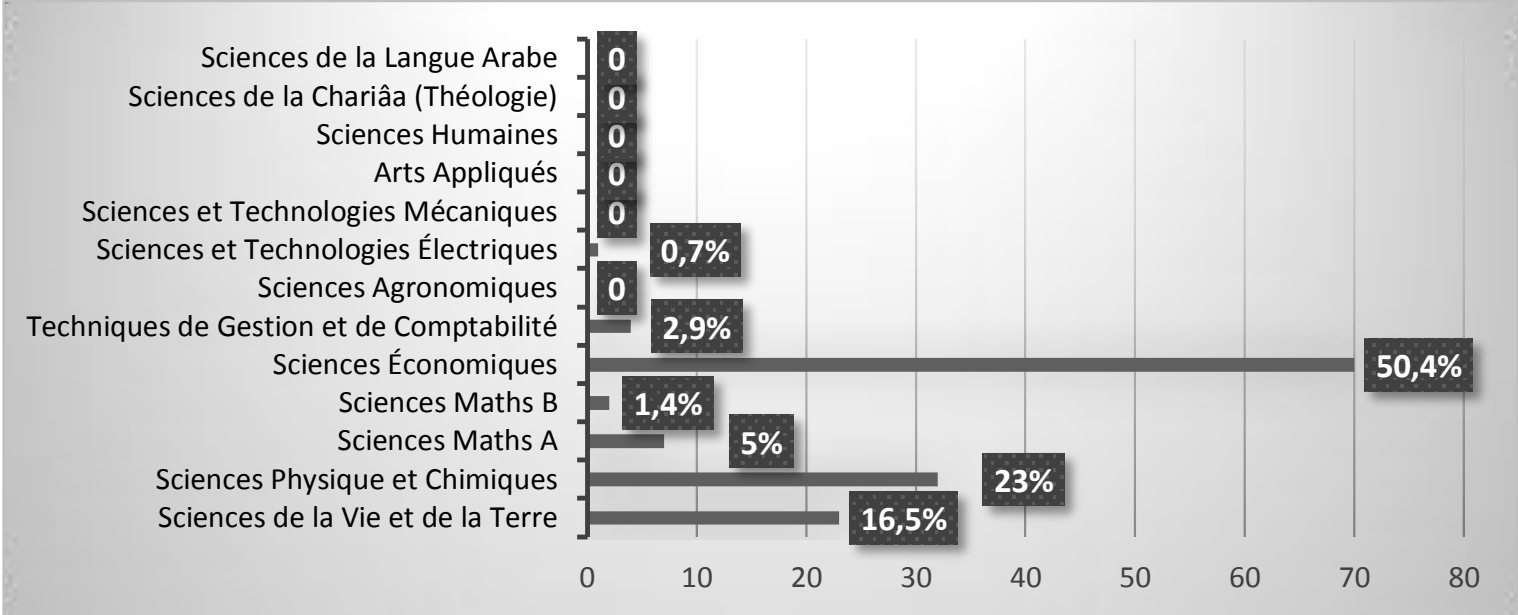

Figure 2:- l'origine scolaire (selon le baccalauréat obtenu)

Une grande majorité des enquêtés sont des bacheliers âgés entre 18 et 20 ans. Quant au type de baccalauréat obtenu, $50,4 \%$ ont suivi des études en sciences économiques, $23 \%$ relèvent de la filière « physique \& chimie » et $16,5 \%$ ont obtenu un baccalauréat option «sciences de la vie et de la terre ». La proportion d'étudiants titulaires d'un baccalauréat option «mathématiques et techniques » est peu significative.

\section{Leur orientation :}

\section{Leurs sources informationnelles}

La famille et les amis $(64,7 \%)$, la recherche sur internet $(45,3 \%)$ et les professeurs de lycée $(23,7 \%)$ sont les trois principales sources auprès desquelles les étudiants s'informent et prennent conseil concernant leur choix d'orientation pédagogique et professionnelle.

D'autre part, et comme sur le schéma ci-après (Figure 3), 28\% des sondés pensent que c'est par le biais de l'université qu'ils vont concrétiser leur projet professionnel, $24 \%$ ont opté pour l'université suite à leur nonadmission en filière sélective (dans un établissement à accès régulé), 14\% sont encouragés par leurs familles à poursuivre leurs études à l'université et $13 \%$ ont choisi l'université en raison tout simplement de sa proximité de leurs domiciles.

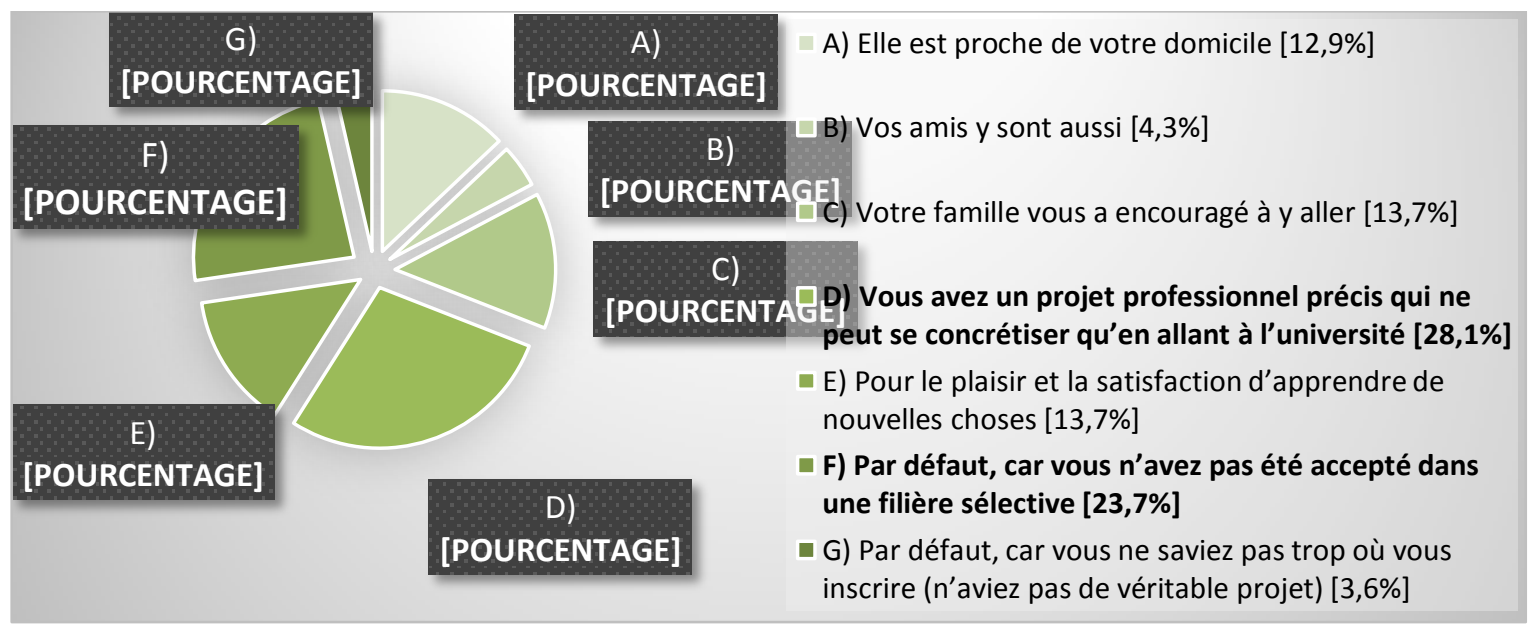

Figure 3:-Les raisons ayant poussé les enquêtés à poursuivre leurs études à l'université 
Les raisons du choix de la filière Sciences économiques et de gestion (SEG) :

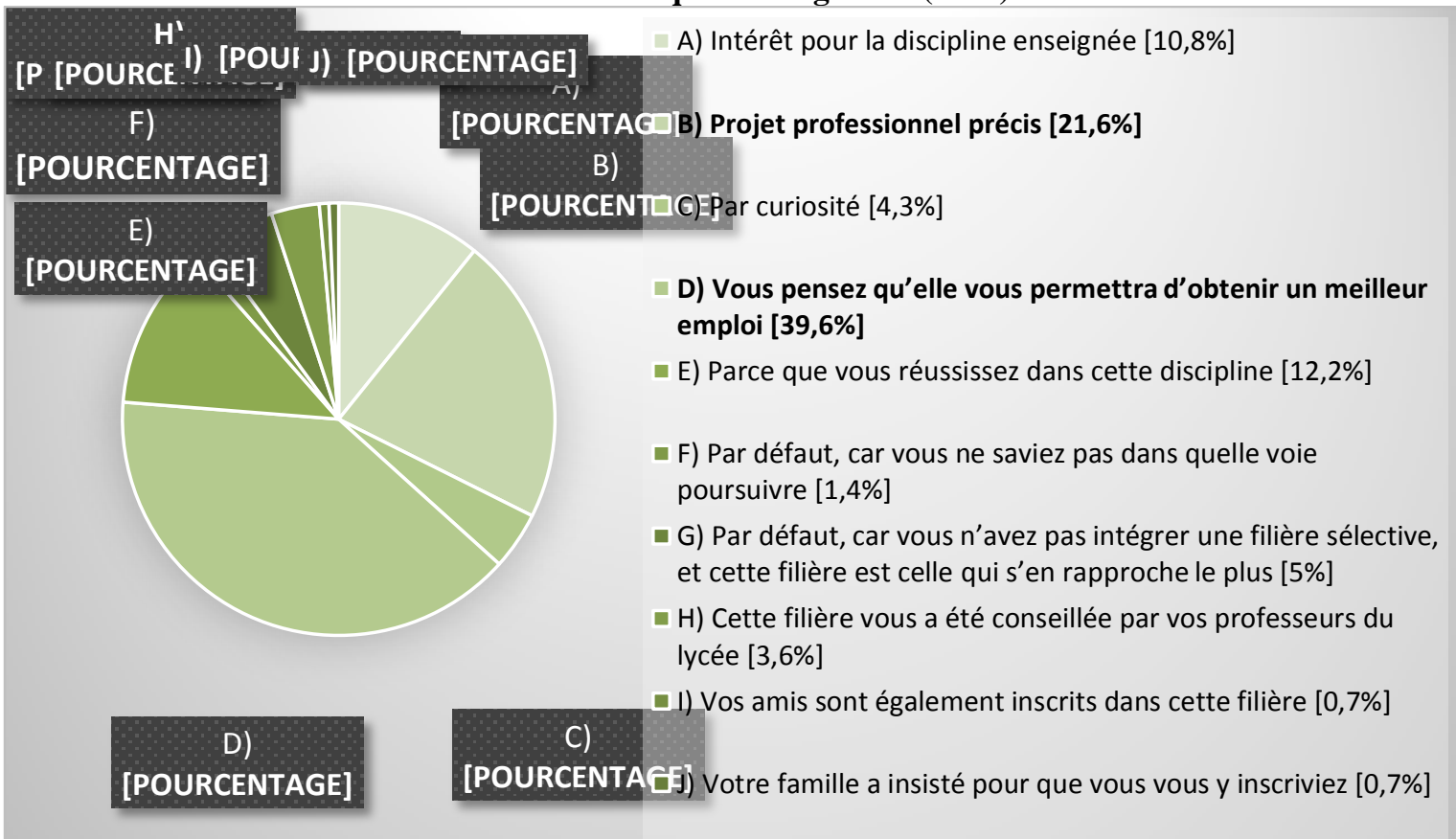

Figure 4:-Raisons du choix de de la filière (SEG)

Le graphique ci-dessus nous renseigne sur les déterminants du choix de la filière lors de l'inscription à l'université. A peu près $40 \%$ ont choisi la filière sciences économiques et de gestion parce qu'ils pensent qu'elle leur permettra d'avoir un meilleur emploi que s'ils choisissent une autre filière, $21,6 \%$ des enquêtés ont déjà un projet professionnel précis concordant avec cette filière et $12 \%$ ont choisi cette filière parce qu'ils l'associent à une réussite et insertion professionnelle aisée.

\section{Leurs projets professionnels}

Il convient de rappeler que tous les enquêtés sont des étudiants ayant suivi les enseignements du PPE et ont eu l'occasion de le réaliser en groupe.

$64,5 \%$ de ces étudiants n'avaient aucune idée sur ce que c'est qu'un projet professionnel avant de suivre les enseignements du PPE et 35,5\% déclarent avoir un projet professionnel précis dès le départ.

Par ailleurs, nous avons regroupé par secteur d'activité professionnelle ou métier les différents projets professionnels décrits par les étudiants et il s'avère qu'ils ont une affinité prononcée pour les métiers de la banque et de la finance (comptabilité et audit), sans oublier les métiers liés au commerce. Enfin, l'envie de créer sa propre entreprise est exprimée par $11,5 \%$.

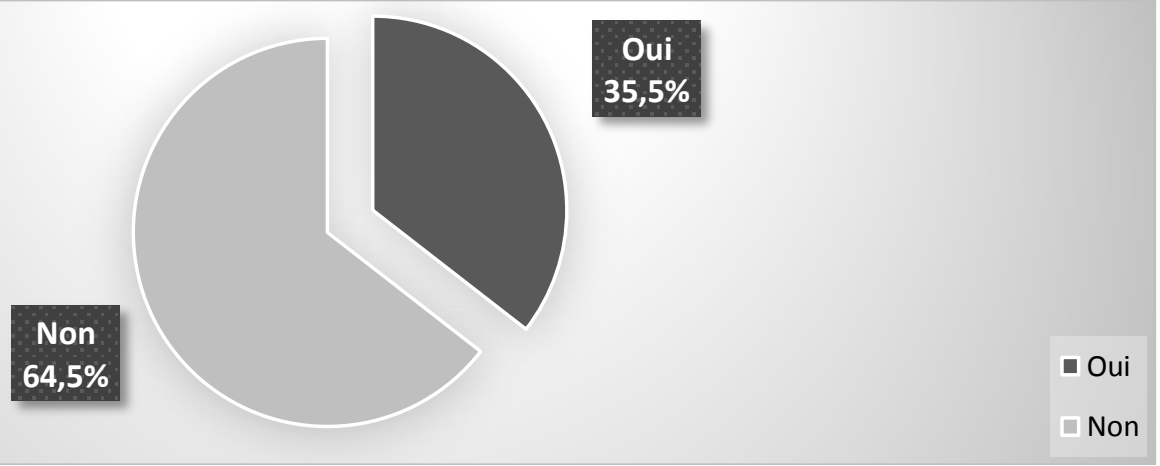

Figure 5:-Aviez-vous un projet professionnel au début du semestre ? 


\section{L'évaluation étudiante du PPE}

Pensez-vous que cet enseignement vous a permis de :

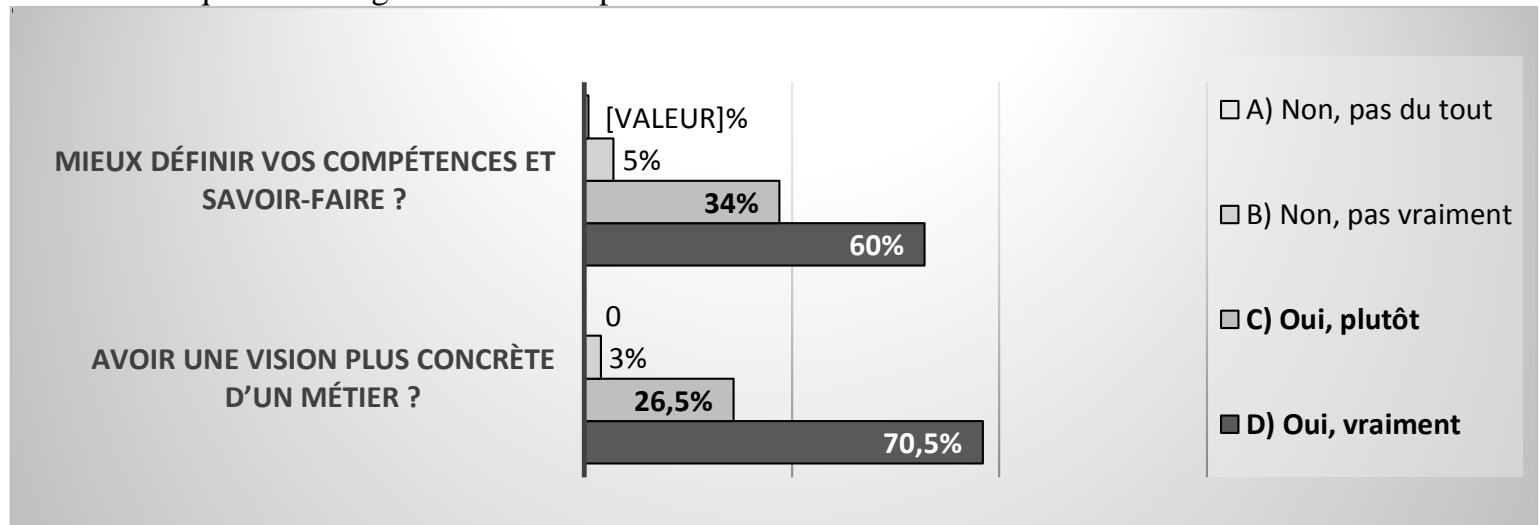

Figure 6:-Impact du cours PPE sur les perceptions de l'étudiant du métier qu'il convoite

L'enseignement du PPE semble atteindre son objectif puisque les enquêtés affirment majoritairement que le PPE leur a permis de mieux percevoir la réalité professionnelle envisagée et les a encouragés à définir et à identifier les compétences clés à consolider.

Le travail demandé dans le cadre de l'enseignement PPE a donné lieu aux avis suivants :

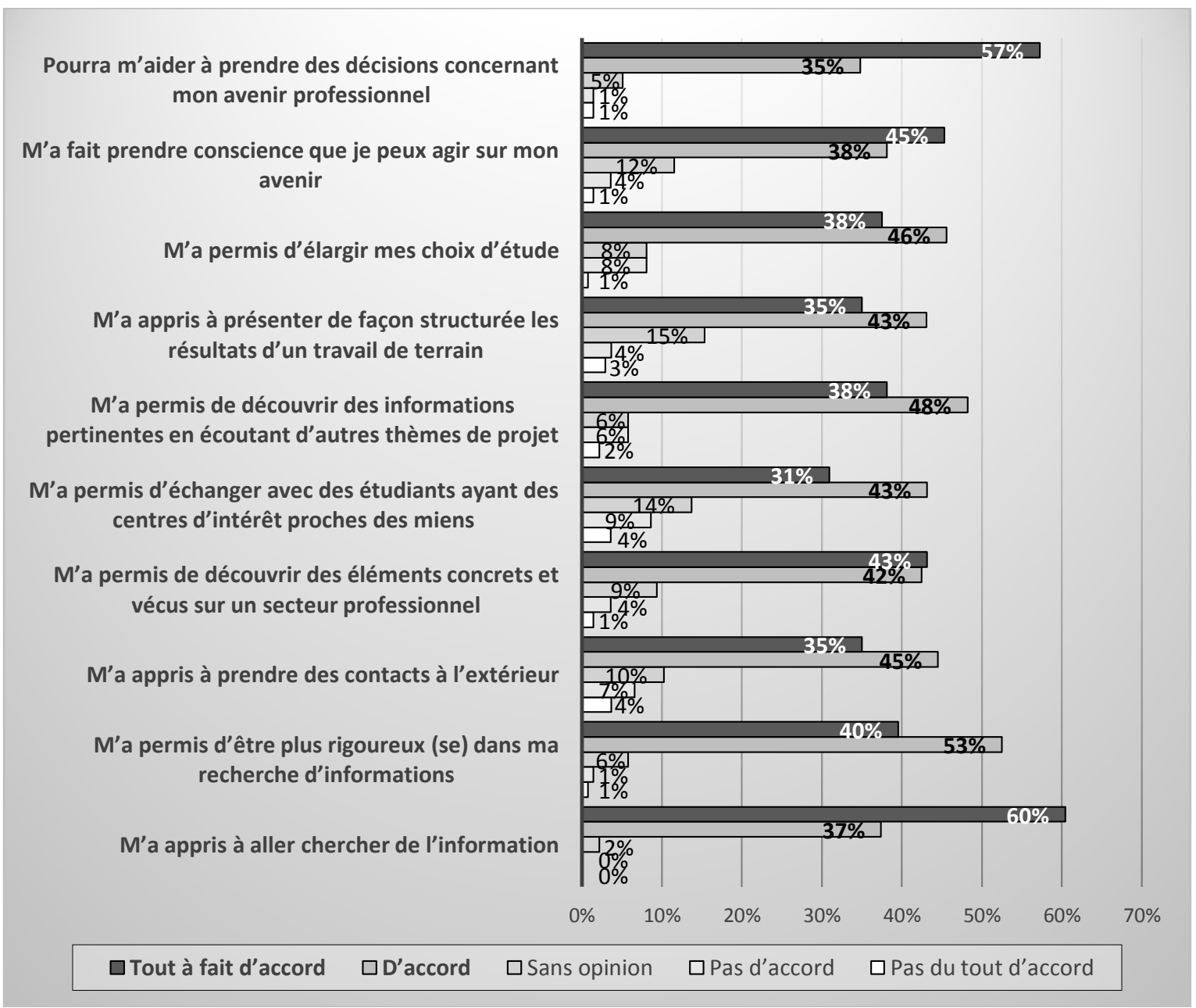

Figure 7:-Les retombées du PPE sur l'étudiant (compétences et savoir-faire) 
Le graphique précédent montre les incidences en termes de compétences développées et/ou mises en œuvre par les étudiants lors de la réalisation de ce PPE.

Ainsi, le PPE leur a permis de s'engager rigoureusement dans la recherche des informations sur le métier convoité. Ils étaient amenés à se déplacer à l'extérieur des murailles de l'université afin de se renseigner notamment auprès des professionnels qui le plus souvent leur ont consacré des entrevues. Certaines d'entre elles ont même été enregistrées, sur demande des étudiants et après consentement du professionnel. Ce qui a permis une plus large diffusion de son message auprès des autres groupes PPE lors des présentations finales.

Le PPE n'était pas seulement une occasion pour l'étudiant de faire son introspection, de se projeter dans l'avenir et de prendre conscience de la réalité professionnelle envisagée, mais également l'opportunité de réfléchir sur les orientations académiques possibles et susceptibles de garantir une meilleure insertion professionnelle à chaque étudiant selon ses atouts, choix, préférences et compétences.

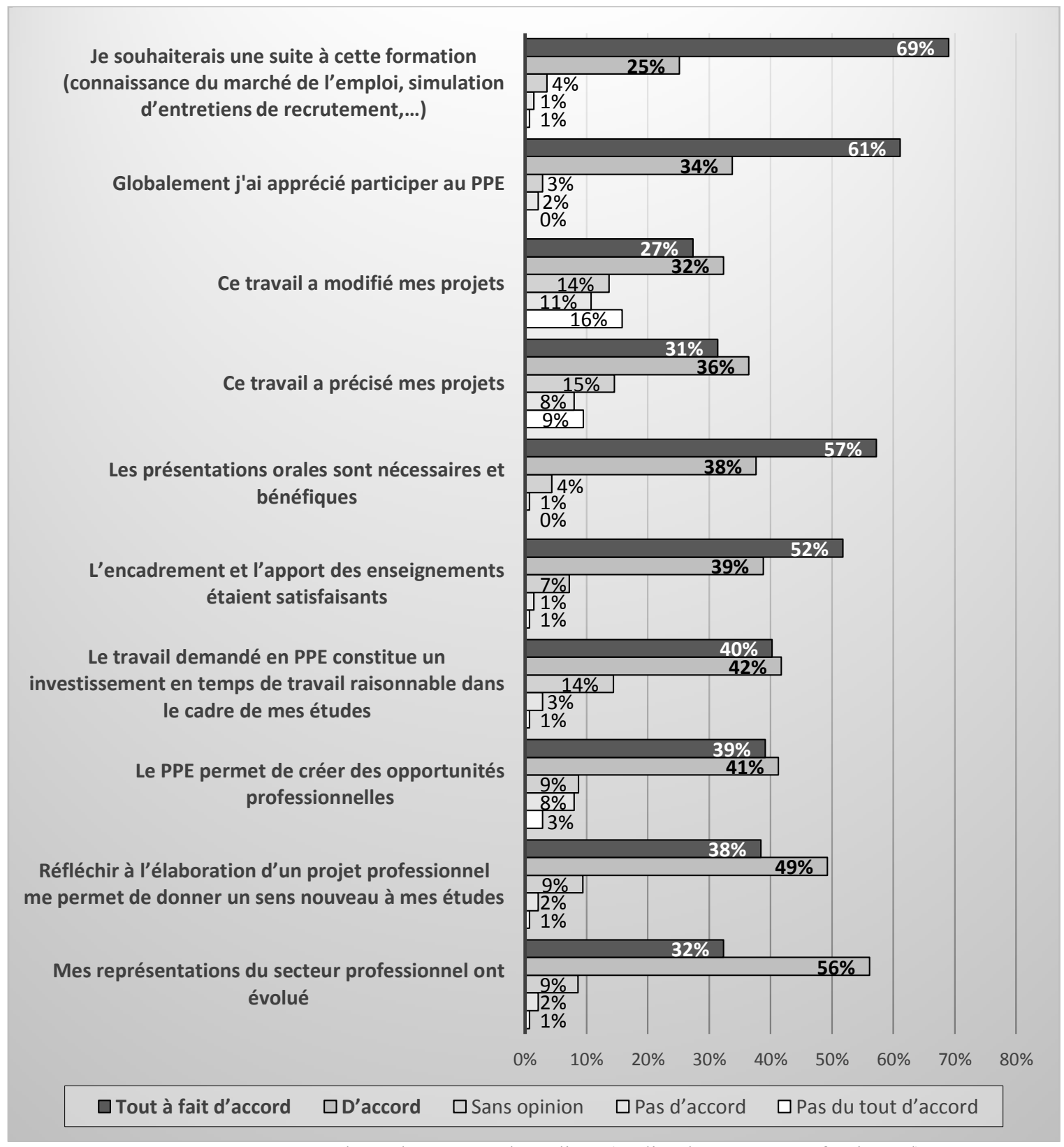

Figure 8:-Les retombées du PPE sur l'étudiant (réalité du secteur professionnel) 
Le graphique ci-dessus résume l'intérêt du dispositif tel que perçu par les étudiants sondés. Globalement, les perceptions du secteur professionnel ont majoritairement évolué chez les étudiants grâce aux recherches d'informations et aux rencontres avec les professionnels (les visites d'entreprises et des administrations publiques en l'occurrence).

Aussi, le PPE leur a permis de développer certaines compétences transversales telles que la prise de parole en public, le travail en équipe et l'écoute active, comme l'illustrait la Figure 8.

\section{Principales conclusions tirées de l'enquête}

Notre étude montre que les objectifs du PPE sont vraisemblablement atteints, et que si les bacheliers se trouvent le plus souvent perdus dans leurs choix d'orientation et finissent majoritairement par choisir leurs études supérieures en fonction de leurs seuls résultats (dans l'enseignement secondaire) et se tournent essentiellement vers leurs familles, amis et professeurs du lycée pour leur prodiguer des conseils (relatifs au choix de parcours académique) aux fins d'intégrer in fine un secteur professionnel porteur. Autrement dit, en fonction essentiellement de leurs résultats et de la représentation propre de ce qu'est un secteur prometteur ou métier d'avenir (synonyme de prospérité) pour ces conseillers improvisés ou "de fortune" (sans que les représentations de ces derniers ne soient nécessairement avérées ou vérifiées dans les faits, le plus souvent), c'est parce qu'il y a absence d'une véritable approche holistique fédérant l'orientation pédagogique, le devenir professionnel, les aspirations et atouts de l'étudiant, ainsi que les pratiques enseignantes.

Le PPE dont les objectifs essentiels (peuvent être ramenés à trois et sont présentés ci-après) a le mérite de pallier (partiellement) cette situation en repositionnant l'étudiant en tant que "sujet-acteur" de son propre destin, éclairé par ses propres recherches effectuées directement sur le terrain auprès des professionnels du secteur qu'il vise.

Repositionnement qui n'est pas sans insuffler un nouvel élan. Une impulsion motivante qui estompe ou réduit, sur son sillage, les doutes qui peuvent subsister quant à l'intérêt des études poursuivies à l'université. Ainsi, et grâce à ce type de démarche et attitude proactive découlant d'une réflexion menée en aval par l'étudiant (à partir de ses observations recueillies directement sur le terrain auprès des professionnels), il gagne en autonomie et se trouve à même de se fixer ses propres objectifs, ceux de son PPE et qui visent justement à :

1. rendre l'étudiant acteur de son propre projet professionnel, tout en le confrontant aux réalités professionnelles ;

2. lui faire acquérir une méthode directement transposable à d'autres situations, telle la recherche de stages ou d'un premier emploi.

3. développer en lui au fur et à mesure de sa formation les compétences nécessaires susceptibles de lui garantir une employabilité future.

Notre questionnaire a révélé par ailleurs qu'une bonne partie des étudiants est amenée à reconsidérer ses souhaits personnels d'orientation et d'"opter tout simplement" pour la filière d'études universitaires (sciences économiques et de gestion dans notre cas) offrant "automatiquement" un poste à la clé, du moins, selon eux et/ou leurs "aînés". Comme semblent le relater près de $40 \%$ de nos sondés, et notamment ceux titulaires parmi eux des baccalauréats : « chimie et physique » et « sciences de la vie et de la terre », entre autres.

Notre enquête a démontré que le fait d'avoir ou de réfléchir en termes de PPE (Projet Professionnel de l'Étudiant), consolide chez ce dernier à la fois la motivation et l'implication dans les études associées à son projet professionnel (son PPE). Nous soulignons donc tout l'intérêt de la programmation d'un tel enseignement dès la première année d'études universitaires.

D'autant plus que les incidences escomptées du PPE sur le raisonnement de l'étudiant, sur son autonomie, l'organisation de ses priorités et la pertinence de ses choix, mais aussi celles sur le développement de ses compétences transversales ou comportementales (ses "soft skills", relatives à la prise de parole en public, à l'esprit d'équipe, au leadership, à la motivation de soi et des autres, à l'écoute et aux techniques de recherche et de traitement de l'information, entre autres) qu'il permet d'acquérir devraient, à en juger par nos résultats d'étude, contribuer pleinement à mettre ses nouveaux bachelier sur la bonne voie et à favoriser leur réussite universitaire et professionnelle.

On peut ensuite s'attendre à une réduction des taux d'échec, du fait de l'augmentation de la motivation, de la prise en considération (systématique) par les étudiants de leurs objectifs professionnels quand ils auront à opérer leurs choix futurs d'orientations universitaires (au terme de la deuxième année du cycle de licence) et à un accroissement 
du sens donné à leurs études, et donc à une consolidation de leur motivation et de leurs chances d'insertion professionnelle.

Sur un plan plus général, au-delà de l'élaboration par l'étudiant des premiers éléments de son projet professionnel, le PPE vise à lui apprendre à affronter l'incertitude, à le préparer à la rencontre du hasard ${ }^{[25]}$ (E. Morin, 1999).

\section{Conclusion:-}

L'avènement de l'ère d'une «transformation pédagogique » restructurant le système éducatif et insufflant ce besoin décisif de repenser ou concevoir «de nouvelles formes d'apprentissage et de nouveaux modes d'enseignement » à même d'assurer sa renaissance, apparait désormais comme la clef de voûte nécessaire à l'évolution de l'enseignement supérieur notamment eu égard aux objectifs de réussite académique des étudiants et de ceux de leur adaptation aux exigences de la vie professionnelle.

Dans cette perspective, l'innovation pédagogique se présente comme une réponse adéquate à une demande express à la fois des enseignants, soucieux d'améliorer et d'optimiser leurs pratiques d'enseignement de bout en bout -et donc pour l'ensemble de la formation- tout en s'engageant dans de nouvelles tâches et de nouveaux rôles de médiation, d'encadrement et d'accompagnement vers une insertion couronnant ainsi la mission d'enseignement. Mais aussi, aux différentes réclamations des employeurs qui exigent une main-d'œuvre qualifiée et/ou des salariés compétents, préparés aux défis du marché de travail.

Partant d'une recherche-action, le présent article a rendu compte d'une expérimentation pédagogique innovante visant à autonomiser les étudiants grâce « à l'expression d'un projet professionnel, [et à] [...] les aider à construire leur devenir dans la profession. » ${ }^{[26]}$.

Notre enquête a démontré que le fait d'avoir ou de réfléchir en termes de PPE (Projet Professionnel de l'Étudiant), consolide chez l'étudiant (de la première année universitaire) à la fois la motivation et l'implication dans les études associées à son projet professionnel (son PPE). Aussi, l'engagement dans la réalisation du PPE contribue au développement de son autonomie, de l'organisation de ses priorités et de la pertinence de ses choix, sans oublier le développement de ses compétences transversales ou comportementales. On peut ensuite s'attendre à une réduction des taux d'échec ou de décrochage, du fait de l'augmentation de la motivation, de la prise en considération (systématique) par les étudiants de leurs objectifs professionnels quand ils auront à opérer leurs choix futurs d'orientations universitaires (au terme de la deuxième année du cycle de licence) et à un accroissement du sens donné à leurs études, et donc à une consolidation de leur motivation et de leurs chances d'insertion professionnelle.

Nous soulignons donc tout l'intérêt de la programmation d'un tel enseignement dès la première année d'études universitaires.

Ainsi, si la mise en œuvre du projet professionnel de l'étudiant laisse entrevoir de nouvelles formes d'ouverture plus centrées sur l'étudiant, sur ses choix d'études et sur son avenir professionnel, son implantation et son développement exigent, quant à eux, un engagement conséquent de la part des enseignants qui accepteront - sans réserve- de repenser leurs pratiques pédagogiques habituelles pour jouer le rôle de médiateurs, d'orientateurs et surtout d'accompagnateurs dans la construction des projets de leurs étudiants. Puisqu'ils seront amenés à réorganiser leurs plannings pour animer des encadrements tantôt en grand groupe, tantôt en sous-groupe, voire un suivi individuel, en sus des corrections de rapports et de fiches métiers issues de la recherche documentaire et des entrevues avec les professionnels. D'autres actions tout aussi importantes, doivent être déployées sur différents niveaux du système universitaire (national, établissement, département, filière, programme de formation), en veillant chaque fois à la cohérence avec la politique globale. En effet, une approche systémique doit prendre en compte les différentes facettes de cette politique holistique, liant stratégie de formation, reconnaissance de l'activité d'enseignement, valorisation et aide au "dépassement de soi" dans cette activité, formation des acteurs, numérique et innovation pédagogique lesquelles demeurent toutes interdépendantes et interagissent les unes sur (ou avec) les autres.

Pour que cette pratique (qu'est l'innovation pédagogique) puisse répondre aux critères d'efficacité pédagogique,

${ }^{25}$ EDGAR M., Les sept savoirs nécessaires à l'éducation du futur, Paris, UNESCO, octobre 1999.

${ }^{26}$ BOUTINET, J.-P., Anthropologie du projet, PUF, 1993. 
l'adaptation de l'environnement de travail (outils, matériel, organisation...) aux besoins de l'utilisateur/de l'enseignant, ou encore "l'ergonomie pédagogique » (un concept dont l'usage peut profiter aux parties prenantes de la pédagogie universitaire) s'impose. Ceci en vue de l'atteinte de cet optimum d'efficacité, d'assurance (sécurité) et de plaisir (confort) et ce pour le plus grand nombre des acteurs d'un environnement pédagogique ${ }^{[27]}$.

Autrement dit, sans le soutien institutionnel, tant au niveau local que national et sans un accompagnement administratif (répartition des groupes et des locaux mis à la disposition) et technique (matériels pédagogiques), les innovations pédagogiques n'induiront jamais ni la création de nouvelles pratiques, ni celle de nouvelles méthodes, et encore moins toutes formes d'externalités positives et pour l'institution, et pour l'enseignant et certainement l'étudiant et ses futurs recruteurs éventuels partenaires des institutions, pour s'arrêter au stade de mode éphémère qui finira par lasser et épuiser l'enthousiasme et la résilience des innovateurs.

\section{Bibliographie:-}

1. Loi organisant l'enseignement supérieur au Maroc: www.enssup.gov.ma/sites/default/files/ETABLISSEMENT-SUP/Dahir_n-1-00-199_du19mai2000.pdf

2. MONCHATRE S., Du métier de sociologue au « genre sociologique » : réflexions sur la professionnalisation. Sociologies Pratiques, ${ }^{\circ} 21$, p. 139-147, 2010.

3. AGULHON C., La professionnalisation à l'université, une réponse à la demande sociale ? Recherches et Formations, $n^{\circ}$ 54. 2007.

4. ROSE J., La professionnalisation des études supérieures, tendances, acteurs et formes concrète. Les chemins de la formation vers l'emploi, Relief, 2008.

5. CORTEEL D., Zimmermann B., Capacités et développement professionnel. Formation Emploi, n $98,2007$.

6. Entretien avec le directeur de l'ANEAQ (28/02/2017) : http://www.aneaq.ma/fr/aneaq_eco.pdf.

7. ABDALLAH F., Meta-modélisation pour décrire et instrumenter une situation d'apprentissage de pédagogie par projet collectif. Thèse de doctorat en informatique. Le Mans : Université du Maine, 2009.

8. PERRENOUD P., Réussir ou comprendre ? Les dilemmes classiques d'une démarche de projet, Genève.1998.

9. PROULX J., Apprentissage par projet, Presses de l'Université du Québec, 2004.

10. LEBRUN M., Théories et méthodes pédagogiques pour enseigner et apprendre : Quelle place pour les TIC dans l'éducation ? (2e éd.). Bruxelles : De Boeck Supérieur. 2007.

11. LE BOTERF G., Compétence et navigation professionnelle. Paris : Éditions d'Organisation. 2000.

12. LATREILlE G., Les chemins de l'orientation professionnelle : trente ans de lutte et de recherche. Lyon : Presses Universitaires de Lyon. 1984.

13. GALAND B., Neuville, S. et Frenay, M., L’échec à l'université en Communauté Française de Belgique : Comprendre pour mieux prévenir? In Galand B. (dir.) : L'échec à l'université en Communauté Française de Belgique (pp. 5-17). Cahiers de recherche en éducation et formation, $\mathrm{n}^{\circ}$ 39. 2005.

14. ROMAINVILlE M. et Parmentier, P., Les manières d'apprendre à l'université. In Frenay M., Noël B., Parmentier P. et Romainville M. (dir.): L'étudiant-apprenant: Grilles de lecture pour l'enseignant universitaire (pp. 63-80). Bruxelles : De Boeck Université. 1998.

15. COULON A., Le Métier d'étudiant : l'entrée dans la vie universitaire. In: Revue française de pédagogie, volume 131. Les formations professionnelles entre l'Ecole et l'Entreprise. pp. 138-139. 2000.

16. BECHARD J.-P. et Pelletier P., Développement des innovations pédagogiques en milieu universitaire : un cas d'apprentissage organisationnel, in Raymond D. (dir.), Nouveaux espaces de développement professionnel et organisationnel, Éditions du CRP, 2001.

17. CHARLOT B., Du rapport au savoir. Éléments pour une théorie. Paris : Anthropos. 1997.

18. LISON C., Bédard D., Beaucher C. et Trudelle D., De l'innovation à un modèle de dynamique innovationnelle en enseignement supérieur. Revue Internationale de Pédagogie en Enseignement Supérieur (RIPES), 30-1, 2014. En ligne : http://ripes.revues.org/771.

19. CROS F. et Adamczewski, G. (dir.), L'innovation en éducation et en formation. Bruxelles, De Boeck.1996.

20. POUMAY M., L'innovation pédagogique dans le contexte de l'enseignement supérieur. In: Lameul G. et Loisy C. (dir.). La pédagogie universitaire à l'heure du numérique (p. 69-81). Louvain-la-Neuve : De Boeck. 2014

21. BECHARD J.-P., L'enseignement supérieur et les innovations pédagogiques : une recension des écrits. Revue des sciences de l'éducation, 272 (2001), 257-281. 2001.

${ }^{27}$ LEPLAT, J., Compétence et ergonomie. In: Amalberti R., De Montmollin M. et Theureau J. (Eds.), Modèles en analyse du travail (pp. 263-278). Liège : Mardaga, 1991. 
22. LISON C et Bédard D., Développement de la posture intellectuelle d'étudiants universitaires dans un programme innovant, Revue internationale de pédagogie de l'enseignement supérieur, 30-1, 2014. En ligne : http://ripes.revues.org/795

23. LATREILLE G., Les chemins de l'orientation professionnelle : trente ans de lutte et de recherche. Lyon : Presses Universitaires de Lyon. 1984.

24. BLAIS M. et Martineau S., L'analyse inductive générale : description d'une démarche visant à donner un sens à des données brutes, Recherches qualitatives, vol. 36, n², 2006.

25. EDGAR M., Les sept savoirs nécessaires à l'éducation du futur, Paris, UNESCO, octobre 1999.

26. BOUTINET, J.-P., Anthropologie du projet, PUF, 1993.

27. LEPLAT, J., Compétence et ergonomie. In: Amalberti R., De Montmollin M. et Theureau J. (Eds.), Modèles en analyse du travail (pp. 263-278). Liège : Mardaga, 1991. 